\title{
Evaluation of liver parenchyma and perfusion using dynamic contrast-enhanced computed tomography and contrast-enhanced ultrasonography in captive green iguanas (Iguana iguana) under general anesthesia
}

\author{
Giordano Nardini ${ }^{* *}$, Nicola Di Girolamo ${ }^{2}$, Stefania Leopardi ${ }^{1}$, Irene Paganelli ${ }^{1}$, Anna Zaghini ${ }^{3}$, \\ Francesco C Origgi ${ }^{4}$ and Massimo Vignoli ${ }^{5}$
}

\begin{abstract}
Background: Contrast-enhanced diagnostic imaging techniques are considered useful in veterinary and human medicine to evaluate liver perfusion and focal hepatic lesions. Although hepatic diseases are a common occurrence in reptile medicine, there is no reference to the use of contrast-enhanced ultrasound (CEUS) and contrast-enhanced computed tomography (CECT) to evaluate the liver in lizards. Therefore, the aim of this study was to evaluate the pattern of change in echogenicity and attenuation of the liver in green iguanas (Iguana iguana) after administration of specific contrast media.

Results: An increase in liver echogenicity and density was evident during CEUS and CECT, respectively. In CEUS, the mean \pm SD (median; range) peak enhancement was 19.9\% \pm 7.5 (18.3; 11.7-34.6). Time to peak enhancement was $134.0 \pm 125.1$ (68.4; 59.6-364.5) seconds. During CECT, first visualization of the contrast medium was at $3.6 \pm 0.5$ (4; 3-4) seconds in the aorta, $10.7 \pm 2.2(10.5 ; 7-14)$ seconds in the hepatic arteries, and $15 \pm 4.5(14.5 ; 10-24)$ seconds in the liver parenchyma. Time to peak was $14.1 \pm 3.4(13 ; 11-21)$ and $31 \pm 9.6(29 ; 23-45)$ seconds in the aorta and the liver parenchyma, respectively.

Conclusion: CEUS and dynamic CECT are practical means to determine liver hemodynamics in green iguanas. Distribution of contrast medium in iguana differed from mammals. Specific reference ranges of hepatic perfusion for diagnostic evaluation of the liver in iguanas are necessary since the use of mammalian references may lead the clinician to formulate incorrect diagnostic suspicions.
\end{abstract}

\section{Background}

Although in reptiles hepatic diseases are traditionally suspected to be secondary to husbandry mismanagement, hepatic diseases caused by parasitic infestations [1], viral [2-4] and bacterial [5,6] infections, and neoplastic proliferations $[7,8]$ are sporadically reported in the literature.

Even though hepatic diseases are quite common in reptiles they are hardly diagnosed ante mortem due to

\footnotetext{
* Correspondence: giordano.nardini@gmail.com

'Veterinary Clinic Modena Sud, Spilamberto, MO 41057, Italy

Full list of author information is available at the end of the article
}

the ambiguous clinical signs $[2,4,5]$. Furthermore, the function of the reptilian liver is markedly influenced by age, sex, physiological condition, temperature, and other environmental conditions [9]. Changes in the organ's size, color, and texture, as well as alterations of biochemical parameters [10] may therefore be alternatively considered either normal or related to hepatic diseases. For example, in chelonians there are physiological fluctuations of liver enzymes that depend on the seasons and on the gender $[11,12]$. Therefore, the diagnosis of liver pathological conditions is challenging and evaluation of the liver through multiple approaches is often necessary. 
In green iguanas (Iguana iguana) an effective endoscopic technique for collection of liver biopsy specimens has been described [13]. Endoscopic biopsy is a powerful technique, especially when dealing with multifocal/ diffuse diseases. Unfortunately, there are no data in the current literature describing the sensitivity of liver endoscopic biopsy when dealing with focal hepatic disease. Although to achieve a definitive diagnosis tissue biopsy may be required, a preliminary characterization of liver parenchyma and perfusion by use of non-invasive imaging techniques may be useful to evaluate the presence of lesions, to localize them and to determine the extent of their distribution.

In companion animal medicine, several techniques allow morphological evaluations of the liver. Among them, ultrasonography has been widely employed, while computed tomography $(\mathrm{CT})$ and contrast-enhanced computed tomography (CECT) are being increasingly utilized [14]. Although ultrasonography provides details on the morphology and the vascularization of the liver, contrastenhanced ultrasound (CEUS), based on the injection of specific contrast agents, allows the investigation of the perfusion of tissues [15]. When a tissue is perfused with an ultrasound contrast agent, analysis of grayscale images collected over an appropriate duration of time permits creation of time-intensity curves for a chosen region of interest. Mathematical analysis of these time-intensity curves yields quantitative hemodynamic indices relating to blood flow in either tissue volumes or within individual vessels [15]. Perfusion parameters are of interest since changes in vascularity and blood flow secondary to pathology generate alterations in the time-intensity curve [16]. Furthermore, contrast-enhanced ultrasound allows a more complete characterization of focal lesions [17].

In canine patients contrast-enhanced ultrasonography appears to improve the characterization of focal and multifocal hepatic lesions [18] and in humans CEUS detected significantly more focal liver lesions than unenhanced ultrasonography [19]. Apart for imaging of the liver $[17,18]$ CEUS has also been demonstrated to be a useful for imaging other organs including canine spleen $[17,20]$, lymph nodes [21], prostate [22,23] kidneys $[24,25]$, and adrenals [26].

Computed tomography may also provide useful clinical information when used to evaluate perfusion of hepatic parenchyma and of focal hepatic lesions $[27,28]$. Although in reptiles CT has been historically used to describe the normal anatomy [29-33], more recently, probably due to the easier access to CT scanners, it has been employed for clinical evaluation of anatomical changes [34,35]. Therefore, description of reference ranges for liver hemodynamics of healthy individuals may be useful to permit assessment of the liver in clinical settings.
Although both CECT and CEUS could be powerful diagnostic tools to evaluate liver morphology and perfusion, to the best of the authors' knowledge, no studies have so far evaluated their use in reptiles. Therefore, the aim of this study was to evaluate architecture and perfusion of the liver in green iguanas (Iguana iguana) by use of CECT and CEUS, to serve as a reference for future clinical studies. The specific hypothesis was that distribution of the contrast media after intravenous injection in a peripheral vein would result in a rise in echogenicity and attenuation of the liver, and therefore CECT and CEUS would be useful tools to evaluate liver perfusion.

\section{Results}

\section{Population summary}

Of eleven iguanas presented in the study period, eight (7 males, 1 female) met the criteria for inclusion in the study. Two iguanas were excluded for clinical abnormalities, and one iguana was excluded due to abnormal values on serum biochemistry. Mean age of the included iguana was 9 years (range 2-18 yr), and mean body weight was $1.9 \mathrm{~kg}$ (range 1.1-2.8 kg). All the iguanas recovered uneventfully from anesthesia and no notable complications were associated with the procedure. Histological examination did not reveal the presence of significant tissue changes except for a mild to moderate degree of hepatic lipidosis detected in all the liver samples observed.

\section{CEUS}

Upon B-mode ultrasonography, the liver was easily visualized in all iguanas (Figure 1A). After the injection of contrast medium (wash in phase, Figure 1B) an increasing echogenicity in the hepatic arteries was initially observed, followed by a more diffuse and homogenous enhancement of the liver parenchyma during the portal phase, until the peak intensity was reached (Figure 1C). During the wash out phase, a homogenous decrease of the echogenicity was visible in all cases (Figure 1D). Complete clearance of the contrast medium was not achieved neither in the 3 individuals in which image acquisition ran for 10 minutes. Representative frames of the scanning sequence after contrast medium administration from one of the iguanas are shown in Figure 1, and a representative time-intensity curve over the region of interest is shown in Figure 2.

The mean peak enhancement was $19.9 \% \pm 7.5$ (18.3; 11.7-34.6). TTP ceus $_{\text {was }} 134.0 \pm 125.1$ (68.4; 59.6-364.5) seconds. Distribution of both peak enhancement and $\mathrm{TTP}_{\text {ceus }}$ were normal and no outliers were detected.

Repeatability of CEUS was adequate, with intraindividual variations of peak enhancement and $\mathrm{TTP}_{\text {ceus }}$ of $10.5 \%$ and $1.2 \%$, respectively. 


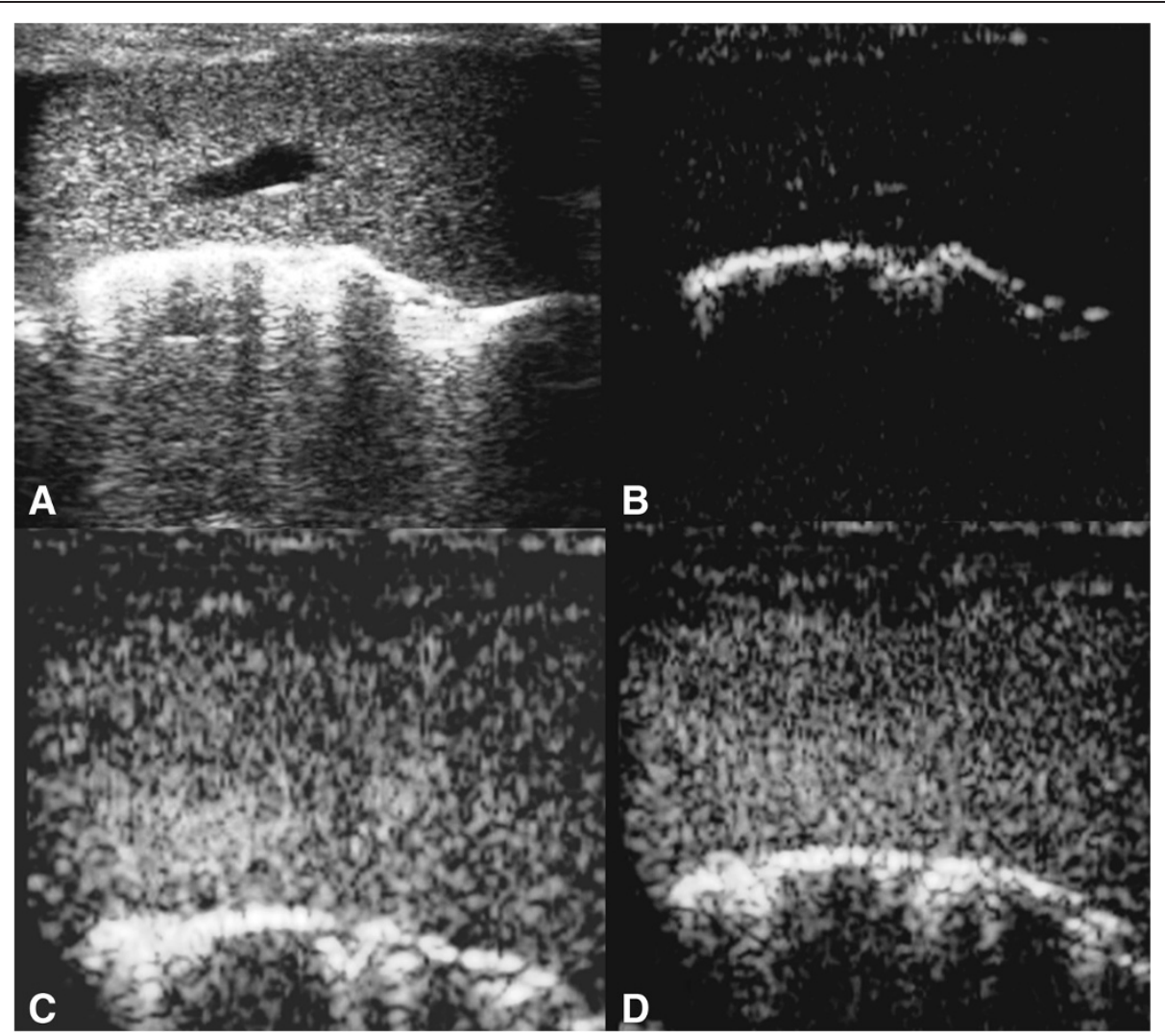

Figure 1 Representative ultrasonographic evaluation of the liver of an iguana included in the study. Artifacts secondary to the scales are visible during B-mode examination (A). At T0, before injection of contrast media, (B) no contrast enhancement of the liver vessels is present, while enhancement it is well visible 36 (C) and 90 (D) seconds after contrast medium injection.

\section{CECT}

Liver parenchyma and margins were easily visualized in the plain CT scan (Figure 3). Basal liver attenuation as measured through CT scan analysis was $77.3 \pm 6.2 \mathrm{HU}$ reaching $179.1 \pm 35 \mathrm{HU}$ at peak enhancement. The first visualization of the contrast medium was at $3.6 \pm 0.5$ (4; 3 -4) seconds in the aorta, $10.7 \pm 2.2(10.5$; 7-14) seconds in the hepatic arteries, and $15 \pm 4.5$ (14.5; 10-24) seconds in the liver parenchyma. $\mathrm{TTP}_{\text {cect }}$ in the aorta was $14.1 \pm$ $3.4(13 ; 11-21)$ seconds. TTP ${ }_{\text {cect }}$ in the liver parenchyma was $31 \pm 9.6(29 ; 23-45)$ seconds. One iguana (No. 8) was found to be an outlier, presenting slower enhancement in aorta (21 seconds) and slower arrival of contrast medium in the liver (24 seconds) than the population studied (Figure 4) and was excluded from the descriptive statistics. A time-intensity curve representative of the population studied is reported in Figure 5.

\section{Discussion}

Results of the present study indicate that imaging techniques employing intravenous distribution of contrast media may be used in green iguanas to evaluate liver texture and perfusion. The distribution of the contrast agent within the liver either during contrast-enhanced ultrasound and contrast-enhanced computed tomography was accompanied by a clearly recognizable change in liver

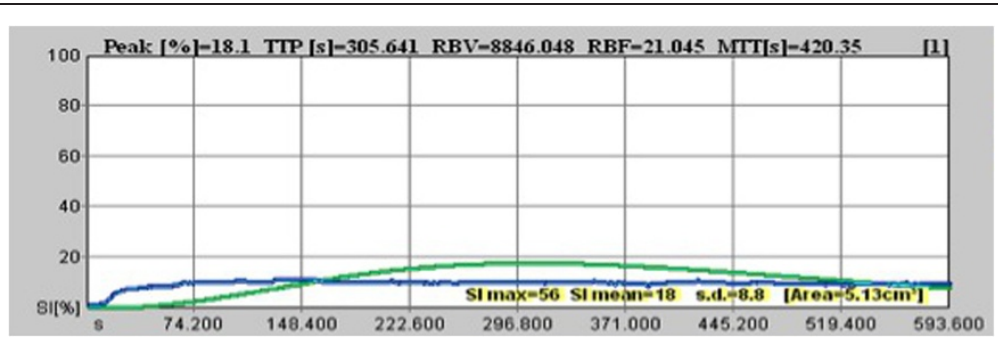

Figure 2 Time-intensity curve of the distribution of SonoVue in the liver of a representative green iguana. 


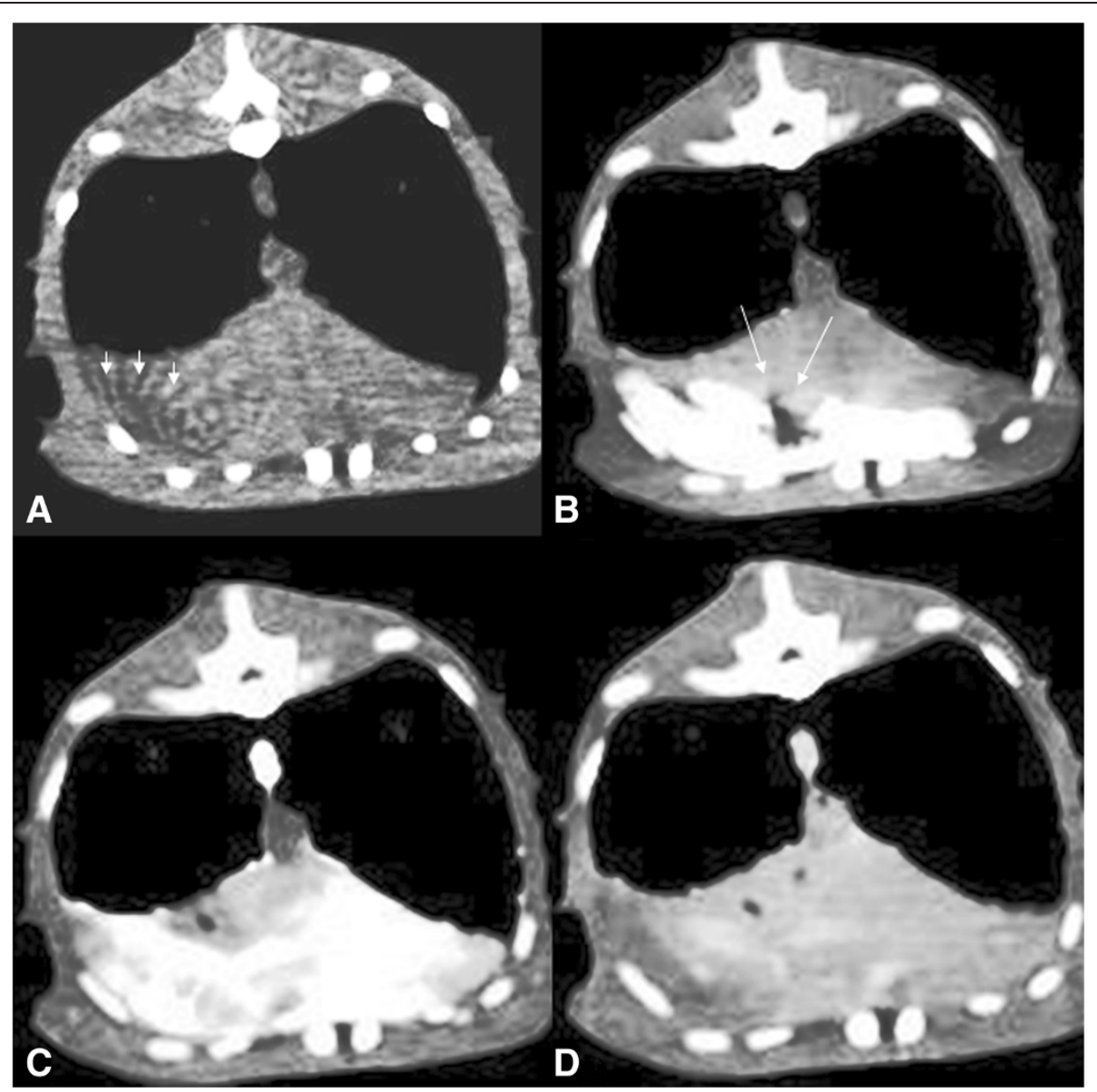

Figure 3 Representative dynamic CT study of an iguana included in the study. No vascular enhancement is present in the vessels just after the contrast medium injection (A); the contrast medium is present in the caudal vena cava, but not in the aorta 3 seconds after injection (B); 36 seconds after injection is well visible in all the liver parenchyma (C), and after 600 seconds from the injection start, the contrast medium it is still mildly appreciable (D). Beam hardening (arrowheads - A) and blooming (arrows - B) artifacts are present due to ribs, and the pool of contrast medium in the caudal vena cava respectively.

echogenicity and liver attenuation, respectively. More invasive techniques, such as endoscopic biopsy [13], have been previously employed to evaluate the liver in green iguanas, but to the authors knowledge, this is the first time that enhancement of liver tissue in iguanas after administration of contrast media during CT and ultrasound examinations is evaluated. Although liver biopsy is considered to be the gold standard for diagnosing hepatic diseases, it may be useful to have reference ranges for diagnostic imaging techniques that permit evaluation of liver hemodynamics.

By use of the same contrast medium employed in the present study, during CEUS examination of the liver in dogs $[15,36]$ a (1) hepatic arterial phase at $10-25$ s postinjection has been defined, followed by a (2) portal vein phase at 20-90 seconds post-injection. This last phase lasted 150-240 seconds. Such phases reflect the double vascularization of the hepatic tissue by the hepatic artery ( $20 \%$ to $30 \%$ of blood) and the portal vein $(70 \%$ to $80 \%$ of blood) [15,37]. In green iguanas we observed a similar pattern, characterized by a (1) increased echogenicity of the hepatic arteries and (2) diffuse enhancement of the liver parenchyma, until the peak intensity was reached. The wash out phase in the iguanas was characterized by a gradual decrease in echogenicity of the liver parenchyma. In all cases enhancement of the liver lasted for more than 10 minutes.

There are two findings of the present study that are worthy of mention, as they strongly differed from what is expected in mammals. Firstly, time to peak, a measure of the time needed to the contrast medium to provide the maximum enhancement of a target organ (ie, the liver), was determined to be 134 (range 59-364) seconds in our study, drastically longer than what is described in dogs and cats. In conscious and anesthetized dogs, time to peak enhancement occurred among 15 to 46 seconds after injection of the contrast media [15,36]. In cats, time to peak occurred approximately 10 seconds after 


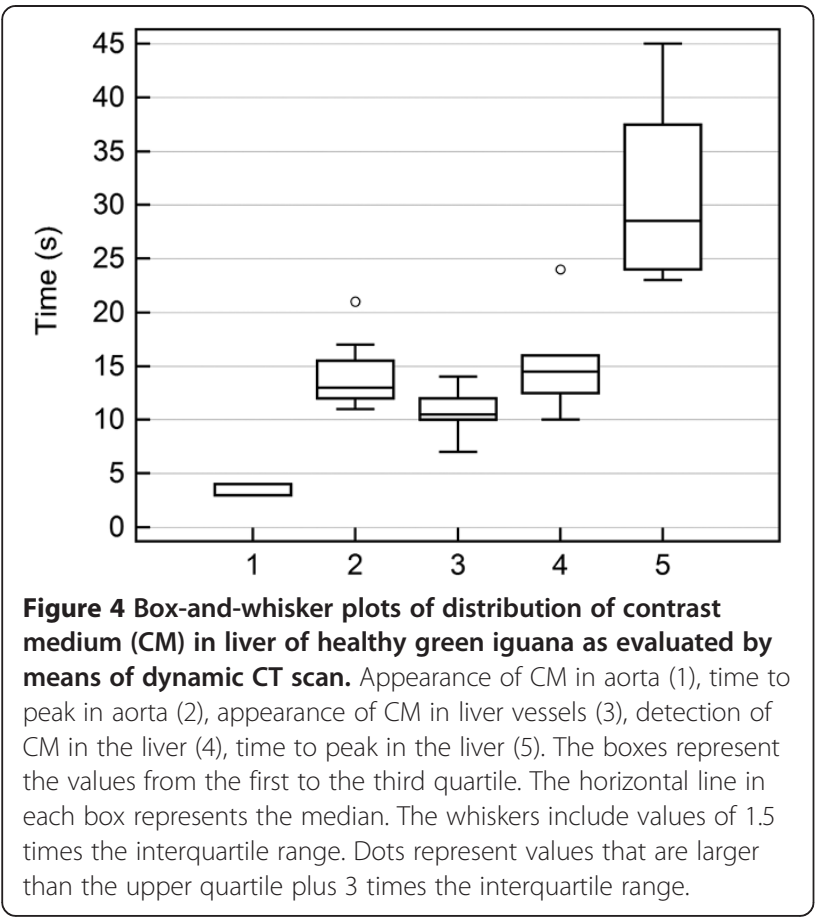

injection of the contrast agent, with a significant enhancement of liver parenchyma from approximately $10 \mathrm{~dB}$ of baseline intensity to approximately $30 \mathrm{~dB}$ of peak intensity [38]. This finding is especially relevant because (1) if results of CEUS examination of green iguanas are interpreted extrapolating current knowledge on dogs and cats, healthy green iguanas would be suspected to suffer a delay in reach of time to peak enhancement. Furthermore, (2) when performing contrast-enhanced imaging techniques to evaluate the liver in lizards, longer studies should be planned than that performed in mammals.
The second unexpected finding was the inter-individual variance observed in time to peak enhancement. In previous studies on dogs and cats, time to peak was characterized by a relatively small standard deviation: in 11 dogs the standard deviation was of 20 seconds (mean 34.6 seconds) and in 10 cats the standard deviations was of 2.9 seconds (mean 9,6 seconds) [36,38]. This suggests that time to peak is a parameter somehow consistent among individuals of the same species. In contrast, in the iguanas studied here $\mathrm{TTP}_{\text {ceus }}$ varied greatly among individuals with a much higher standard deviation (over 100 seconds). Due to the design of our study it is impossible to objectively determine which factor was responsible for such high inter-individual variability. We suppose that the greater variance for time to peak observed in this study may be secondary to intrinsic factors (eg, vitellogenesis, large variation in the age of the iguanas), or to the use of chemical restraint. In fact, although all the iguanas underwent the same anesthetic protocol, effect of anesthetic agents has historically been considered to show much greater variability among reptiles that in mammals [39].

In the present study all the examinations have been performed in iguanas under general anesthesia. Although in aggressive iguanas chemical restraint may be necessary to perform CEUS safely, in most occasions manual restraint should be sufficient to perform a standard CEUS examination of the liver in clinical setting. To facilitate the examination the stimulation of the vaso-vagal response, via light digital pressure applied to the eyelids, could be performed [40]. Nevertheless, it should be taken in account that results obtained in the present study may not apply to conscious iguanas or iguanas in which the baroreceptor reflex has been stimulated: In a previous study evaluating CEUS of the liver in dogs, time to peak enhancement was

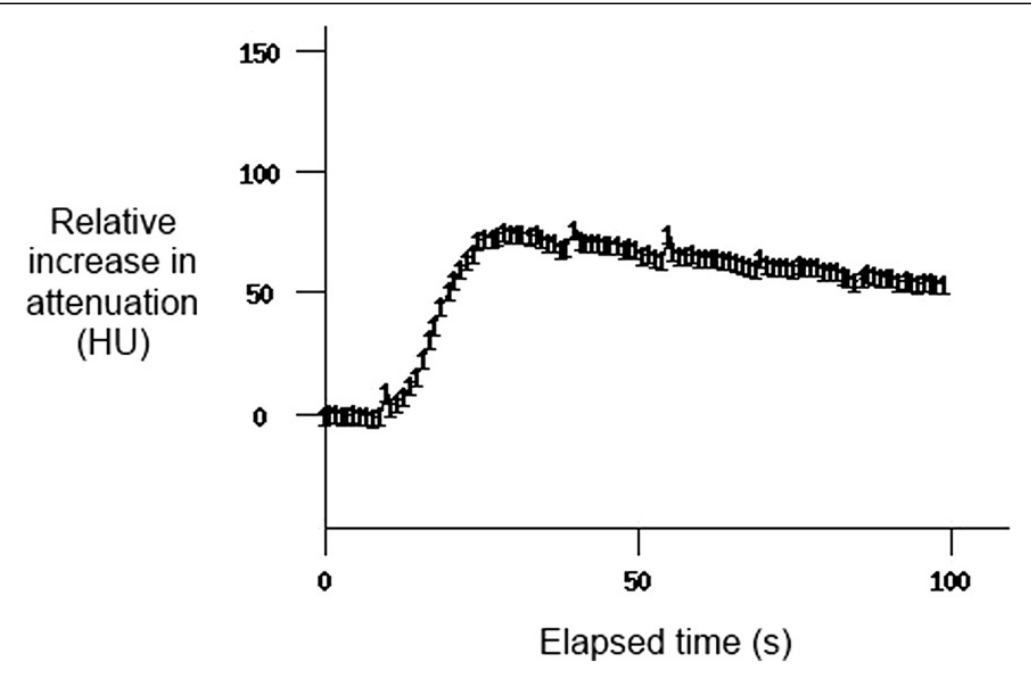

Figure 5 Time-density curves for aorta and liver during 600 seconds of investigation in a representative iguana. 
significantly shorter when dogs were anesthetized with propofol [36]. Such difference was suspected to be secondary to the effect that propofol has on the vascular system (ie, increase in hepatic arterial blood flow and decrease of systemic arterial pressure [41]). In any case, the other parameters did not differ in conscious and anesthetized individuals. Therefore, when considering the values produced in our study, the use of anesthetic drugs has to be considered, especially concerning time to peak enhancement.

Unenhanced CT scans provided excellent visualization of lizard's liver. Contrast-enhanced computed tomography permitted further investigation of perfusion. Time-density curves obtained in iguanas were not dissimilar to curves that are usually obtained in mammals [42]. The graphic obtained for the aorta was characterized by a very pendant curve during wash-in with a high peak, and an initial rapid decrease followed by a slower second phase during washout. In the liver the wash-in phase was slower and the peak lower, whereas the pattern after peak was similar to that of the aorta, both of which did not complete washout during the 600 seconds of investigation. The curve pattern appeared quite similar in all animals studied apart from a few differences noted in the graphs for iguana No. 8. The distribution of the contrast medium in Iguana No. 8 was characterized by slower $\mathrm{TTP}_{\text {cect }}$ in aorta and slower visualization of contrast medium in the liver, resulting in an evident right shifting of the time-density curve. In all the individuals studied the contrast medium was still partially visible in the aorta and in the liver after a 600 seconds period of investigation. Such slower wash out phase compared to mammals [36] is probably caused by the lower metabolic rates of reptiles, which averages $25 \%$ to $35 \%$ of that of mammals [43].

Minimal fluctuations visualized in some of the timeintensity curves were probably associated with the respiratory acts of the iguanas. Although difficulties on keeping the ROI in the middle of the scanned area of the liver is reported also in anesthetized cats [38], we suspect that in reptiles, the fluctuations secondary to the relation between lung size and pressure in visceral organs may be more relevant due the absence of a muscular diaphragm.

In the present study absolute values for mean gray level were reported only when strictly necessary, as these values may be affected by several variables such as gain setting, mechanical index, scanning depth, the size and body composition of the individual animal, and the behavior of the individual contrast medium [36]. Changes in attenuation of the liver are for example described in chelonians, in which hypoattenuating liver (ie, $<20$ Hounsfield Units) was associated with hepatic lipidosis [44]. Considering that a moderate grade of liver steatosis was present in the individuals studied, it is possible to hypothesize that iguanas without any degree of steatosis may present a more attenuating liver parenchyma.
Another factor that should be taken in account, whenever the present study is used as a reference, is that the contrast medium was injected into the ventral tail vein. The other reasonable vascular accesses in iguanas are the cephalic vein and the jugular vein, although they usually require a surgical incision of the skin [45]. Some differences in time to peak enhancement may be expected if injection of the contrast medium is performed in a different vein due to the different endovascular transit done by the contrast medium.

Proper cohort or case-control studies in iguanas would be ideal to identify an increased risk in mortality or in adverse events in animals undergoing CEUS or CECT [46]. Such typology of studies is rarely performed in reptile medicine due to the overall limited number of reptile patients, and to the multitude of confounding factors that should be considered (eg, species, metabolism, housing). Nevertheless, based on the lack of complications in the present study and based on evidence recently acquired in dogs and cats [47], there is no indication to suppose that these techniques are harmful.

\section{Conclusions}

Normal liver attenuation and perfusion were determined by evaluation of the contrast medium diffusion in the parenchyma. Time-density curves of the liver were characterized by a fast time to peak and a slow wash out which was not completed during the recorded time. An important inter-individual variation is present in clinically healthy iguanas. However, due to the small population sampled in this study we cannot determine whether this is a consistent phenomenon in this species.

Further studies in iguanas with hepatic diseases are needed in order to evaluate potential differences in the attenuation between normal and abnormal livers. In particular, inclusion of lizards with focal hepatic lesions would be aimed, as characterization of focal lesions is one of the most powerful applications of contrast-enhanced imaging techniques. Lastly, future studies including captive and wild iguanas may allow evaluation of whether the moderate lipidosis observed in the hepatic samples was secondary to housing or dietary conditions.

\section{Methods}

\section{Animals}

Clinically healthy, client-owned, captive-born green iguanas (Iguana iguana) presented to the Clinica Veterinaria Modena Sud (Spilamberto, MO, Italy) in a three-month period were eligible for inclusion in the study. To be included in the study iguanas needed to have no previous pathologies reported and to be maintained in proper dietary (ie, fed mixtures of leafy green vegetables and had access to water ad libitum) and housing conditions (ie, presence of a basking spot reaching $35^{\circ} \mathrm{C}\left[95^{\circ} \mathrm{F}\right]$, 
UVB lamps replaced at least biannually, exposure to natural sunlight in spring and summer). The animals were considered healthy on the basis of physical examination and of clinical biochemistry values within published reference ranges $[48,49]$. Biochemistry was performed by means of a bench-top analyzer (VetScan, Abaxis, Inc., Union City, CA) that use commercially available rotor designed to be used in Avian/Reptilian patients. Parameters analysed for each animal were albumin, aspartate transaminase, biliary acid, calcium, creatine kinase, glucose, phosphorus, potassium, sodium, total protein, and uric acid.

The study was performed in compliance with the directive 2010/63/EU of the European parliament and of the European council. The institutional ethical committee of the University of Bologna approved all the procedures. The owners gave written informed consent for the enrolment of their animals in the study.

\section{Procedures}

The ventral tail vein of each iguana was catheterized using a 22 gauge IV catheter (Jelco, Smiths Medical International Ltd, Lancashire, UK) inserted at two-thirds of the tail length. Anesthesia was induced with a slow intravenous $10 \mathrm{mg} / \mathrm{kg}$ [4.54 mg/lb] injection of propofol (Fresenius Kabi, Isola della Scala, Italy) [50] with the animals maintained in a warm room $\left(28^{\circ} \mathrm{C}\left[82.4^{\circ} \mathrm{F}\right]\right)$ for 24 hours before the procedure. Iguanas were intubated with non-cuffed tracheal tube (with diameter between 2.0 and $3.0 \mathrm{~mm}$ ) and connected to a closed Y pediatric circuit. Anesthesia was maintained administering 2.0\% isoflurane and 0.8-1.2 litre/minute oxygen through an adjustable dial (concentration range, 0-5\%) coupled with a separate oxygen flow meter (range, 0.2-4 L/min). Manual ventilation was performed if apnea lasted more than 20 seconds. The heart rate was monitored through a doppler probe placed on the jugular vein during the entire procedure. Ultrasound and CT scans were performed as described below. Following the imaging session ultrasound-guided liver biopsies were obtained from each iguana. After the procedures the iguanas were individually placed in a small warm enclosure $\left(32^{\circ} \mathrm{C}\left[89.6^{\circ} \mathrm{F}\right]\right)$ and closely monitored during recovery from anesthesia.

\section{Ultrasonography procedures}

A survey liver scan was performed using standard B-mode ultrasonography with $5-7.5 \mathrm{MHz}$ linear transducer with coded harmonic capability (Esaote Mylab 30, Esaote-CnTI System, Esaote, Genova, Italy), to ensure there were no visible liver lesions, and to permit selection of a suitable acoustic window, i.e., one that provided an uninterrupted view of as large a section of liver parenchyma as possible. The animals were maintained in ventro-dorsal position, and large amount of gel was used in order to reduce artifacts caused by entrapment of air bubbles in between the scales.

A second-generation contrast agent composed of sulphur hexafluoride microbubbles (SonoVue $8 \mathrm{mcl} / \mathrm{ml}$, Bracco Imaging S.p.A., Milan, Italy) and a dedicated contrastenhanced ultrasound analytical software (Contrast Tuned Imaging, Contrast Tuned Imaging technology, Esaote, Genova, Italy) were used. When an appropriate acoustic window was found, a rapid bolus dose of $0.03 \mathrm{ml} / \mathrm{kg}$ of the contrast medium was injected through the IV catheter followed by a rapid bolus of $1.5 \mathrm{ml}$ of saline $(0.9 \% \mathrm{NaCl})$. The timer was activated at the moment of the injection $(\mathrm{T}=0)$ and the flow of contrast into the liver was observed in real-time and digitally recorded for 1 minute and 30 seconds. On the first 5 iguanas two consecutive CEUS examinations were performed 30 minutes apart to assess repeatability of the method. As results of the first 5 examinations showed that in 1.5 minutes there was no clearance of the contrast medium in the liver, recording was prolonged to 10 minutes.

Videos were analyzed using specific software (Qontrast, Esaote, Italy) to generate time-intensity curves for each exam. The peak enhancement and time to peak ( $\left.\mathrm{TTP}_{\text {ceus }}\right)$ were calculated for each individual. Perfusion parameters were defined as follows: peak enhancement (maximum signal intensity reached during the transit of the bolus, expressed in \% where $100 \%$ means the maximum), $\mathrm{TTP}_{\text {ceus }}$ (time of arrival of contrast agent post-injection to its maximum peak enhancement value).

\section{CT procedures}

Dynamic CT images were obtained by use of a multidetector 16 slices CT scanner (BrightSpeed 16, GE Corporate, Milwaukee, WI) with the animals being in ventral recumbence. After the plain images were taken, a section of the liver close to the hilum was chosen and one image was taken every second starting from the injection time for a total of 600 images, in order to obtain curves of liver perfusion enhancement. A dose of $800 \mathrm{mg} / \mathrm{kg}$ of an iodinated contrast medium (Ioversol $320 \mathrm{mg} / \mathrm{ml}$, Optiray, Covidien Spa, Italy) [42], was injected trough the ventral tail vein, at $3 \mathrm{ml} / \mathrm{sec}$, using a power injector (Optistar injector, Mallinckrodt plc, Dublin, Ireland).

Liver attenuations in the plain studies and at peak enhancement were determined. Time of first visualization of contrast media in the aorta, in the hepatic arteries, and in the liver parenchyma was recorded. Times to peak $\left(\mathrm{TTP}_{\text {cect }}\right)$ in aorta and in the liver parenchyma were also determined by visual inspection of the curves.

\section{Histopathological procedures}

A biopsy device (Spirotome $10 \mathrm{G}$, Medinvents NV, Hasselt, Belgium) composed by cutting cannula, trocar, helical tissue receiving needle, and releasing device with a 
cut length of $18 \mathrm{~mm}$ was used to obtain liver biopsies in the iguanas, according to the technique previously described in companion animals [51]. A small cutaneous incision was performed with a number 11 scalpel blade in the iguanas to facilitate the introduction of the device through the skin. The biopsies were taken both from the right and the left lobe, distant from any visible vessel. A total of 16 biopsies (two per each animal), were collected by a board-certified radiologist (MV).

Tissue samples were immediately placed into $2 \mathrm{ml}$ screw-top plastic tubes containing 10\% buffered formalin. The tissues were then routinely processed and stained with hematoxylin and eosin. Tissue sections were examined by a board-certified veterinary pathologist (FCO).

\section{Statistical analysis}

Statistical analysis was performed by use of a commercial software (MedCalc 12.2.1, MedCalc Software, Mariakerke, Belgium). Data are reported as mean \pm SD (median; range) unless otherwise stated. P-values less than 0.05 were considered significant. Non-normality was investigated for each parameter through the D'Agostino-Person test. Tukey method was employed to detect outliers, i.e., values smaller than the lower quartile minus 1.5 times the interquartile range, or larger than the upper quartile plus 1.5 times the interquartile range. Repeatability of $\mathrm{TTP}_{\text {ceus }}$ and CEUS peak enhancement was measured calculating the CV from duplicate measurement [52].

\section{Competing interests}

The authors disclose any financial interests with companies that manufacture products that are the subject of the present research or with companies that manufacture competing products.

\section{Authors' contribution}

GN, AZ and MV conceived the study. GN cared for the iguanas, performed the venous catheterisation, analysed the images and assisted in drafting the manuscript. ND analyzed the data and drafted the manuscript. SL assisted with the anesthesia of the iguanas and wrote a first draft of the manuscript IP assisted with the ultrasonographic procedures. FCO analyzed the liver biopsies. MV performed the diagnostic examinations, performed the liver biopsies, analyzed the images and assisted in drafting the manuscript. All authors read and approved the final manuscript.

\section{Acknowledgments}

The study was partially funded by the Associazione Benessere Animale (ABA), Modena.

\section{Author details \\ ${ }^{1}$ Veterinary Clinic Modena Sud, Spilamberto, MO 41057, Italy. ${ }^{2}$ Clinica per Animali Esotici, CVS, Rome 00137, Italy. ${ }^{3}$ Department of Veterinary Medical Sciences, University of Bologna, Ozzano dell'Emilia, BO 40064, Italy. ${ }^{4}$ Center for Fish and Wildlife Health (FIWI), College of Veterinary Medicine, University of Bern, Vetsuisse Faculty, Bern, $\mathrm{CH} 3012$, Switzerland. ${ }^{5}$ PetCare Veterinary Association, Marzabotto, BO 40043, Italy.}

Received: 15 January 2014 Accepted: 1 May 2014

Published: 13 May 2014

\section{References}

1. Richter B, Csokai J, Graner I, Eisenberg T, Pantchev N, Eskens HU, Nedorost $\mathrm{N}$ : Encephalitozoonosis in two inland bearded dragons (Pogona vitticeps). J Comp Path 2013, 148:278-282.

2. Hughes-Hanks JM, Schommer SK, Mitchell WJ, Shaw DP: Hepatitis and enteritis caused by a novel herpesvirus in two monitor lizards (Varanus spp.). J Vet Diagn Invest 2010, 22:295-299.

3. Moormann S, Seehusen F, Reckling D, Kilwinski J, Puff C, Elhensheri M, Wohlsein P, Peters M: Systemic adenovirus infection in bearded dragons (Pogona vitticeps): histological, ultrastructural and molecular findings. J Comp Pathol 2009, 141:78-83.

4. Wilkinson M, Cline M, Jerome WG: Cytopathic herpesvirus infection in a green iguana (Iguana iguana). J Zoo Wildl Med 2005, 36:724-726.

5. González Candela M, Martín Atance P, Seva J, Pallarés FJ, Léon Vizcaíno L: Granulomatous hepatitis caused by Salmonella Typhimurium in a spur-thighed tortoise (Testudo graeca). Vet Rec 2005, 157:236-237.

6. Angus KW: Granulomatous hepatitis in tortoises. Vet Rec 2005, 157:328.

7. Martorell J, Ramis A, Espada Y: Use of ultrasonography in the diagnosis of hepatic spindle-cell sarcoma in a savannah monitor (Varanus exanthematicus). Vet Rec 2002, 150:282-284

8. Knotek Z, Dorrenstein GM, Hrda A, Tomek A, Proks P, Knotková Z, JekI V, Lewis W: Hepatocellular carcinoma in a green iguana - a case study. Acta Vet Brno 2011, 80:243-247.

9. Schaffer F: The Liver. In Biology of the Reptilia. Volume 19th edition. Edited by Gans C, Gaunt AS. St Louis: Society for the Study of Amphibians and Reptiles; 1998:485-531.

10. Lawrence K: Seasonal variation in blood biochemistry of long-term captive Mediterranean tortoises (Testudo graeca and T hermanni). Res Vet Sci 1987, 43:379-383.

11. Scope A, Schwendenwein I, Schauberger G: Characterization and quantification of the influence of season and gender on plasma chemistries of Hermann's tortoises (Testudo hermanni, Gmelin 1789). Res Vet Sci 2013, 95:59-68.

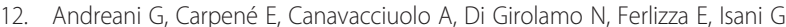
Hematology, plasma biochemistry and protein electrophoresis of Hermann's tortoises (Testudo hermanni ssp.). Vet Clin Path 2014, in press.

13. Hernandez-Divers SJ, Stahl SJ, McBride M, Stedman NL: Evaluation of an endoscopic liver biopsy technique in green iguanas. J Am Vet Med Assoc 2007, 230:1849-1853.

14. Irausquin RA, Scavelli TD, Corti L, Stefanacci JD, DeMarco J, Flood S, Rohrbach BW: Comparative evaluation of the liver in dogs with a splenic mass by using ultrasonography and contrast-enhanced computed tomography. Can Vet J 2008, 49:46-52.

15. Nyman HT, Kristensen AT, Flagstad A, McEvoy FJ: A review of the sonographic assessment of tumor metastases in liver and superficial lymph nodes. Vet Radiol Ultrasound 2004, 45:438-448.

16. Albrecht T, Blomley MJ, Cosgrove DO, Taylor-Robinson SD, Jayaram V, Eckersley R, Urbank A, Butler-Barnes J, Patel N: Transit-time studies with levovist in patients with and without hepatic cirrhosis: a promising new diagnostic tool. Eur Radiol 1999, 9:S377-S381.

17. Rossi F, Leone VF, Vignoli M, Laddaga E, Terragni R: Use of contrastenhanced ultrasound for characterization of focal splenic lesions. Vet Radiol Ultrasound 2008, 49:154-164.

18. Nakamura K, Takagi S, Sasaki N, Bandula Kumara WR, Murakami M, Ohta H, Yamasaki M, Takiguchi M: Contrast-enhanced ultrasonography for characterization of canine focal liver lesions. Vet Radiol Ultrasound 2010, 51:79-85.

19. Dietrich CF, Kratzer W, Strobel D, Danse E, Fessl R, Bunk A, Vossas U, Hauenstein K, Koch W, Blank W, Oudkerk M, Hahn D, Greis C: Assessment of metastatic liver disease in patients with primary extrahepatic tumors by contrastenhanced sonography versus CT and MRI. World J Gastroenterol 2006, 12:1699-1705.

20. Rossi F, Rabba S, Vignoli M, Haers H, Terragni R, Saunders JH: B-mode and contrast-enhanced sonographic assessment of accessory spleen in the dog. Vet Radiol Ultrasound 2010, 51:173-177.

21. Wang Y, Cheng Z, Li J, Tang J: Gray-scale contrast-enhanced ultrasonography in detecting sentinel lymph nodes: an animal study. Eur J Radiol 2010, 74:e55-59.

22. Russo M, Vignoli M, England GC: B-mode and contrast-enhanced ultrasonographic findings in canine prostatic disorders. Reprod Domest Anim 2012, 47:238-242. 
23. Russo M, Vignoli M, Catone G, Rossi F, Attanasi G, England GC: Prostatic perfusion in the dog using contrast-enhanced Doppler ultrasound. Reprod Domest Anim 2009, 44:334-335.

24. Wei K, Le E, Bin JP, Coggins M, Thorpe J, Kaul S: Quantification of renal blood flow with contrast-enhanced ultrasound. J Am Coll Cardiol 2001 37:1135-1140.

25. Haers H, Vignoli M, Paes G, Rossi F, Taeymans O, Daminet S, Saunders JH: Contrast harmonic ultrasonographic appearance of focal spaceoccupying renal lesions. Vet Radiol Ultrasound 2010, 51:516-22.

26. Pey P, Vignoli M, Haers H, Duchateau L, Rossi F, Saunders JH: Contrastenhanced ultrasonography of the normal canine adrenal gland. Vet Radiol Ultrasound 2011, 52:560-567.

27. Zwingenberger AL, Shofer FS: Dynamic computed tomographic quantitation of hepatic perfusion in dogs with and without portal vascular anomalies. Am J Vet Res 2007, 68:970-974.

28. Taniura T, Marukawa K, Yamada K, Hikasa Y, Ito K: Differential diagnosis of hepatic tumor-like lesions in dog by using dynamic CT scanning. Hiroshima J Med Sci 2009, 58:17-24.

29. Arencibia A, Rivero MA, De Miguel I, Contreras S, Cabrero A, Orós J: Computed tomographic anatomy of the head of the loggerhead sea turtle (Caretta caretta). Res Vet Sci 2006, 81:165-169.

30. Maisano JA, Kearney M, Rowe T: Cranial anatomy of the spade-headed amphisbaenian Diplometopon zarudnyi (Squamata, Amphisbaenia) based on high-resolution x-ray computed tomography. J Morphol 2006, 267:70-102.

31. Rieppel $\mathrm{O}$ : The naso-frontal joint in snakes as revealed by high-resolution X-ray computed tomography of intact and complete skulls. Zool Anz 2007, 246:177-191.

32. Valente ALS, Cuenca R, Zamora M, Parga ML, Lavin S, Alegre F, Marco I: Computed tomography of the vertebral column and coelomic structures in the normal loggerhead sea turtle (Caretta caretta). Vet J 2007 174:362-370.

33. Banzato T, Selleri P, Veladiano IA, Martin A, Zanetti E, Zotti A: Comparative evaluation of the cadaveric, radiographic and computed tomographic anatomy of the heads of green iguana (Iguana iguana), common tegu (Tupinambis merianae) and bearded dragon (Pogona vitticeps). BMC Vet Res 2012, 8:53.

34. Preziosi R, Diana A, Florio D, Gustinelli A, Nardini G: Osteitis deformans (Paget's disease) in a Burmese python (Python molurus bivittatus) - a case report. Vet J 2007, 174:669-672.

35. Hall NH, Conley K, Berry C, Farina L, Sigler L, Wellehan JF Jr, Roehrl MH, Heard D: Computed tomography of granulomatous pneumonia with oxalosis in American alligator (Alligator mississippiensis) associated with metatarhzium anisopliae var anisopliae. J Zoo Wildl Med 2011, 42:700-708,

36. Nyman HT, Kristensen AT, Kjelgaard-Hansen M, McEvoy FJ: Contrast-enhanced ultrasonography in normal canine liver. Evaluation of imaging and safety parameters. Vet Radiol Ultrasound 2005, 46:243-250.

37. Haers $\mathrm{H}$, Saunders $\mathrm{JH}$ : Review of clinical characteristics and applications of contrast-enhanced ultrasonography in dogs. J Am Vet Med Assoc 2009, 234:460-470.

38. Leinonen MR, Raekallio MR, Vainio OM, Ruohoniemi MO, Biller DS, O'Brien RT: Quantitative contrast-enhanced ultrasonographic analysis of perfusion in the kidneys, liver, pancreas, small intestine, and mesenteric lymph nodes in healthy cats. Am J Vet Res 2010, 71:1305-1311.

39. Mosley CA, Dyson D, Smith DA: Minimum alveolar concentration of isoflurane in green iguanas and the effect of butorphanol on minimum alveolar concentration. J Am Vet Med Assoc 2003, 222:1559-1564.

40. Smith D, Dobson H, Spence E: Gastrointestinal studies in the green iguana: technique and reference values. Vet Radiol Ultrasound 2001, 42:515-20.

41. Wouters PF, Van de Velde MA, Marcus MA, Deruyter HA, Van Aken H: Hemodynamic changes during induction of anesthesia with eltanolone and propofol in dogs. Anesth Analg 1995, 81:125-131.

42. Zwingenberger AL, Schwarz T: Dual-phase CT angiography of the normal canine portal and hepatic vasculature. Vet Radiol Ultrasound 2004, 45:117-124.

43. Donoghue S: Nutrition. In Reptile Medicine and Surgery. 2nd edition. Edited by Mader DR. St. Louis: Saunders Elsevier; 2006:251-298.

44. Gumpenberger M: Computed Tomography. In Medicine and Surgery Of Tortoises And Turtles. Edited by McArthur S, Wilkinson R, Meyer J. Oxford: Blackwell; 2004:235-238.
45. Mitchell M: Therapeutics. In Reptile Medicine and Surgery. 2nd edition. Edited by Mader DR. St. Louis: Saunders Elsevier; 2006:631-664.

46. Grimes DA, Schulz KF: An overview of clinical research: the lay of the land. Lancet 2002, 359:57-61.

47. Seiler GS, Brown JC, Reetz JA, Taeymans O, Bucknoff M, Rossi F, Ohlerth S, Alder D, Rademacher N, Drost WT, Pollard RE, Travetti O, Pey P, Saunders JH, Shanaman MM, Oliveira CR, O'Brien RT, Gaschen L: Safety of contrastenhanced ultrasonography in dogs and cats: 488 cases (2002-2011). J Am Vet Med Assoc 2013, 242:1255-1259.

48. Divers SJ, Redmayne G, Aves EK: Haematological and biochemical values of 10 green iguanas (Iguana iguana). Vet Rec 1996, 138:203-205.

49. Harr KE, Alleman AR, Dennis PM, Maxwell LK, Lock BA, Bennett RA, Jacobson ER: Morphologic and cytochemical characteristics of blood cells and hematologic and plasma biochemical reference ranges in green iguanas. J Am Vet Med Assoc 2001, 218:915-21.

50. Bennett RA, Schumacher J, Hedjazi-Haring K, Newell SM: Cardiopulmonary and anesthetic effects of propofol administered intraosseously to green iguanas. J Am Vet Med Assoc 1998, 212:93-98.

51. Vignoli M, Barberet V, Chiers K, Duchateau L, Bacci B, Terragni R, Rossi F, Saunders JH: Evaluation of a manual biopsy device, the 'Spirotome', on fresh canine organs: liver, spleen, and kidneys, and first clinical experiences in animals. Eur J Cancer Prev 2011, 20:140-145.

52. Jones R, Payne B: Clinical Investigation and Statistics In Laboratory Medicine. London: CB Venture Publications; 1997.

doi:10.1186/1746-6148-10-112

Cite this article as: Nardini et al:: Evaluation of liver parenchyma and perfusion using dynamic contrast-enhanced computed tomography and contrast-enhanced ultrasonography in captive green iguanas (Iguana iguana) under general anesthesia. BMC Veterinary Research 2014 10:112.

\section{Submit your next manuscript to BioMed Central and take full advantage of:}

- Convenient online submission

- Thorough peer review

- No space constraints or color figure charges

- Immediate publication on acceptance

- Inclusion in PubMed, CAS, Scopus and Google Scholar

- Research which is freely available for redistribution
C BioMed Central 